\title{
Preregistration and Transparency in the Research Process
}

\author{
David Mellor \\ Direct of Policy, Center for Open Science \\ https://orcid.org/0000-0002-3125-5888, @EvoMellor
}

Submitted as part of the AERA 2021 Annual Conference; Session: Increasing the Credibility of Educational Research; SIG-Research Use Paper and Symposium Sessions. Session Chair: Scott Joseph Peters, University of Wisconsin

\section{Table of Contents}

Abstract

Introduction

Rationale and Resources for Authors

Resources and Recommendations for Reviewers

Rationale and Resources for Editors

Being the Change

\section{Abstract}

By submitting a detailed study and analysis plan to a registry prior to conducting a study, scientists can increase transparency into the research lifecycle while making a better distinction between planned, confirmatory hypothesis tests and unplanned, exploratory research. This paper will introduce preregistration as a method as well as share some of the research cited above regarding impact. Resources for preregistering studies will be shared as will be suggestions for how to respond to common concerns or barriers.

\section{Introduction}

Preregistration is one means by which the scientific community can add clarity to the research process. By submitting a detailed study and analysis plan to a registry prior to conducting a study, scientists can increase transparency into the research lifecycle, clarify the amount of ongoing work (regardless of how many results are disseminated via publication), address biases towards statistically significant findings, and make a better distinction between planned, 
confirmatory hypothesis tests and unplanned, exploratory research. Both modes of research are important. However, without a clear distinction, one can unintentionally present results of exploratory analyses using statistical tools designed for a-priori hypothesis testing, which invalidates their diagnostic value. Furthermore, it presents results as being more surprising and publishable, at the expense of their credibility.

Preregistration is appropriate for hypothesis testing studies and for those where methodological decisions, such as variable construction or inclusion rules, can influence inferences. While some study designs present particular challenges for preregistration, such as longitudinal studies or those that rely on existing datasets, the benefits of transparency, better planning, and increased clarity between confirmation and discovery still adds value to the research.

Additional measures to increase the evidential value of reported findings include data, code, and materials sharing, so that the complete research lifecycle can be better evaluated. This transparency increases the impact of reported findings, their credibility, and is particularly useful for original authors, who are better able to quickly adapt well curated materials for their own reuse.

Without preregistration, the scientific literature is prone to bias and exaggeration. The bias for statistically significant research findings is well known (Dwan, Gamble, Williamson, Kirkham, \& the Reporting Bias Group, 2013), but it is also true that this bias produces a scientific literature that is unrepresentative of the total credible evidence. Franco, Malhotra, and Simonovits (2014) found that published research results were unrepresentative of the entire collected evidence even when null results were generated using common methodology. Preregistration can help researchers add credibility to their results, which are more likely to present credible null results (Kaplan \& Irvin, 2015) or more modest effect sizes than when preregistration is not used (Dechartres, et al., 2016; O’Boyle, Banks, \& Gonzalez-Mulé, 2017; Schäfer and Schwarz, 2019).

The combination of "credible" and "null" is particularly important in the preceding paragraph. Measuring two variables that were picked out of a hat and seeing no relationship is to be expected. Conducting an experiment that includes random assignment into groups and seeing no effect on a desired outcome could be expected if the intervention was performed in a sloppy manner. However, that same level of skepticism disappears once a statistically significant finding is reported, which is particularly troubling when one considers the many ways that an 
analysis can lead to such a finding. Take, for example, a study in which 27 teams of analysts analyzed the same research dataset while investigating the same research question (in this case, does a player's skin tone affect the likelihood of receiving a penalty card in soccer) and found 27 different answers to that question (Silberzahn et al., 2018).

However, preregistration is not a magic bullet. Preliminary evidence by Clasen and colleagues (2019) found that the first 27 preregistered studies published in Psychological Science deviated from their analysis plans without complete disclosure. This mirrors lessons learned from clinical trials, where outcome switching still regularly occurs (Goldacre et al., 2019).

There are several key takeaway lessons from these and similar studies that find shortcomings in reporting the results of preregistered research.

First, these shortcomings are only detectable when a preregistration is created. Being able to discover these changes adds value to the research evidence by providing more context and transparency into the research lifecycle. By being able to detect that new outcomes were added, the reader is better able to couch the effectiveness of any given intervention or study procedure. Without preregistration, not only is there no way to detect such changes, but there would be no expectation that such changes were at all problematic. And we know that these practices occur. Self-reported rates of "questionable research practices" (a.k.a. QRPs, or simply poor practices) that invalidate tools of statistical inference are surprisingly high across multiple disciplines, including education research, psychological science, ecology, and evolutionary biology (Agnoli et al., 2017; Fraser et al., 2018; John et al., 2012; Makel et al., 2019). In each of these surveys, a majority of respondents admitted to one or more practices, such as reporting post-hoc tests as if they had been hypothesized prior to analyzing the data, or selectively reporting only results that were statistically significant. The fact that these practices are so widespread and widely admitted to likely stem from both ignorance of the effects these practices have on the credibility of findings, and the clear incentives to report those results that are most publishable.

The second consideration is how a discipline should respond to such shortcomings. As with other practices under the "open science" umbrella, being able to uncover a shortcoming could be used as grounds of rejection, therefore punishing the individual or team for their action. This is especially problematic in an era when such practices as data sharing or preregistration are not required- or when protections for publication (ie through a Registered Reports model of publishing) are not widely implemented. This is why such efforts to improve research culture 
must be proactive, supportive of the individual, paired with editorial/reviewer/reader education efforts, and complemented by improvement in policies toward expecting more transparency as the default.

The onus is on the research community to gain experience with the process of preregistration and complete reporting. Reviewers and authors must come to expect complete reporting of outcomes by not penalizing transparent changes to research designs. This shift in expectation must occur in tandem with increased expectation for preregistration when making inferential claims. Below are specific guidelines for key players to undertake so that, collectively, the community can respond appropriately to changing expectations and increased rigor.

\section{Rationale and Resources for Authors}

A primary benefit of preregistration for authors is to clarify the planned study and outcomes prior to seeing how they will affect the results. This helps to plan the forthcoming research and align general research questions with specific analytical tests. Later, the author will be able to follow the plan in a way that simplifies the later key analysis work. Importantly, preregistration is not a "prison" that would prevent the curious researcher from looking for other key explanations. Far from it, in fact. The plan that is outlined ahead of time can speed one through the hypothesis tests that were a primary rationale for the work and lead one to the, potentially, more interesting discoveries in the dataset. Such a pipeline to these unplanned analyses can be freeing, as one looks for moderators or other explanations for differences in the populations of interest. Instead of "torturing" the data and finding a way to present a compelling story, one is freed to be honest with what works, what doesn't work as expected, and what promising early evidence may be worth following up on.

There are many ways to create a preregistration, but a general recommendation is to start with the default template or guidelines for whichever registry you think works best. Two that are worth highlighting for this audience are the The Registry of Efficacy and Effectiveness Studies (REES) (https://sreereg.icpsr.umich.edu/sreereg/) and the Open Science Framework (https://osf.io ${ }^{1}$ ). The recommended template that is applicable to many studies, both observational and experimental, is the OSF Prereg template (see https://osf.io/preprints/metaarxiv/epgjd/). Searching each registry can help provide some

\footnotetext{
${ }^{1}$ Note that I am an employee of the Center for Open Science (https://cos.io), which builds and maintains the open source OSF.
} 
guidance on how to respond to any questions in particular that may be challenging. However, during an education campaign in which we encouraged adoption of this workflow through $\$ 1,000$ prizes for publishing the results of preregistered work (see the Prereg Challenge: https://www.cos.io/blog/prereg-challenge-ending-whats-next), we anecdotally noticed that through practice came great improvement (which should not be a surprise to anyone!)- authors who submitted 2, 3, or more preregistrations greatly improved the clarity of subsequent plans.

The workflow of course entails both creating the preregistration and then faithfully reporting the results of the preregistered work. In that writeup, a few key principles will guide the results and discussion of the preregistered research. These tips include:

1. Point to the preregistration.

a. By providing a clear URL or DOI to the preregistered work, you help readers, reviewers and editors to follow the planned and conducted work.

2. Report the results of all pre-specified analyses.

a. A reader should be easily led through the hypotheses, the analysis for each one, and their results. If there were 20 prespecified tests, it should be quick and easy to count 20 results.

3. Note any changes from the original plan.

a. Nothing ever goes precisely as expected. That is fine. Disclosing those differences will add credibility to the entire body of work. Consider using a template for reporting such "transparent changes" (e.g. https://osf.io/yrvcg/)

4. Clearly distinguished between the planned analysis, and any unplanned exploratoraiton.

a. Using clear headings, such as Confirmatory and Exploratory Results, can lead the reader to quickly understand how the work was conducted and how it should be interpreted.

By following these simple steps, one can follow the intention of the practice- which is to make clearer distinctions between planned hypothesis tests and serendipitous discoveries.

\section{Resources and Recommendations for Reviewers}

Reviewers play an essential and powerful role in scholarly communication. And as Peter Parker would remind us, with great power comes great responsibility. There is a key opportunity for peer review to make a substantial contribution to the role of open science in academia. Simple 
questions to ask and principles to bear in mind can push authors in the right direction and remind ourselves about the important areas to focus on.

\section{Questions to ask authors}

As a reviewer, one needs to enforce norms within a field; norms that are derived from our own expectations about what makes for credible science. As is widely accepted but often not seen in one's peers, an emphasis on open sharing of evidence and minimizing the influence of irrelevant factors such as dogmatic subscription to explanations is highly valued in the scientific community (Anderson et al., 2007). Reviewers can correct this by explicitly asking for the items we'd like to see- deposition of raw data (or an explanation of which data are accessible and which data have been specially protected), sharing of research materials, curation of code, disclosure of whether or not the work was preregistered. Importantly, reviewers are not policy makers or even decision makers on individual articles- editors remain key players in those two domains. But reviewers can expect that these statements be made clearly in articles and are well within our rights to ask for them. The Peer Reviewer Openness Initiative provides specific recommendations for such statements; and it is very fair to modify those expectations with tasks that one is comfortable asking in their roles as peer reviewers.

\section{Principles to bear in mind when reviewing}

As with the power to ask for specific information in the peer review process, reviewers should remind themselves about the culture we want to set. In particular, transparency allows for better error detection. Especially when such transparency is the exception and not the rule, the consequences for these decisions should not be punitive. We would be harming the move toward better science if the result of this error detection is less transparency with the next article submission after learning a tough lesson. Instead, the peer review process can encourage correction of any errors detected, and discourage reliance on statistical significance or results on the recommendation to reject or accept. Ideally, the process will formalize that decision making framework through adoption of Registered Reports (see below), but even when reviewing a traditional article, ask yourself if the research question was important, if the study was well executed, if others in the field would learn from this evidence. Do not ask if the results are exciting, novel, unexpected, or potentially impactful, as these markers incentivize cherry picking of evidence and reward p-hacking. 


\section{Rationale and Resources for Editors}

Perhaps the most visible steps that can occur to make preregistration a norm in the research community are the responsibility of journal editors. The three specific actions that editors can take include:

1. Adoption of the Registered Reports publishing model

2. Use "Level 2" of the Transparency and Openness Promotion Guidelines standards on preregistration with analysis plans

3. Train reviewers and authors on expected practice

The Registered Reports publishing model (https://cos.io/rr) is a two step process in which proposed studies are evaluated for their importance and rigor prior to observing the outcomes of a study (Nosek \& Lakens, 2014). Essentially, the preregistration is peer reviewed and, if the proposed study is interesting for the community and rigorous in its proposed methods, the outcome will be published regardless of the main result of the study. There are checks that reviewers can insist be included prior to final publication (ie. quality control steps that demonstrate the competence of the research team), but the main outcome of the study cannot influence the final publishability of the study. Resources exist for journals to adopt this format, including suggested language for authors and reviewer instructions (https://osf.io/pukzy/), guidelines for editors (https://osf.io/jbeus/), and implementation checklists (https://osf.io/2m4ct/).

The Transparency and Openness Promotion Guidelines (TOP https://cos.io/top; Nosek et al., 2015) provide specific recommendations for all open science practices. But in particular, they provided recommended steps for supporting preregistration. TOP is tiered to lower barriers to adoption while still providing concrete recommendations for growth and improvement. Level 2 in particular is useful for preregistration. Briefly, this standard states that articles must disclose whether preregistration with an analysis plan exists, and, if so, allows journal access during peer review for verification (the complete recommended policy language is available at https://osf.io/9f6gx/wiki/Guidelines/). This "Level 2" approach is worth adopting because it requires each author to assert whether or not their work was preregistered (as is expected by

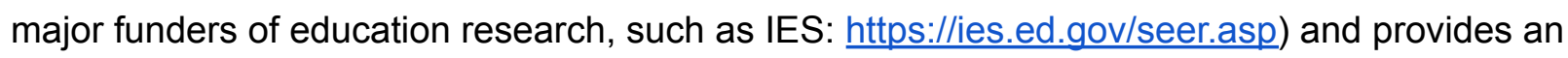
opportunity to verify that that plan was conducted in accordance with the author recommendations mentioned above. Importantly, this standard does not force authors to preregister who are unable or unwilling to do so, or whose methodology is not conducive to that 
process, but it does enable that determination to occur openly. Providing signals that the work was preregistered can be another way to showcase when this work happens (e.g. with open science badges: $\underline{\text { https://cos.io/badges). }}$

\section{Being the Change}

Taking the first step toward adopting open science practices can be daunting. It is helpful to try it out in a safe, sandbox environment prior to conducting it for real. Take a look at various templates available for preregistration (https://osf.io/zab38/wiki/home/) and start doing it when a study presents itself. New standards are being developed for preregistration when research relies on existing datasets (https://osf.io/x4gzt/), uses qualitative methods (Haven et al., 2020), longitudinal studies (https://osf.io/d5b89/), or systematic reviews (https://osf.io/785sb/).

\section{Literature Cited}

Anderson, M. S., Martinson, B. C., \& De Vries, R. (2007). Normative Dissonance in Science: Results from a National Survey of U.S. Scientists. Journal of Empirical Research on Human Research Ethics: An International Journal, 2(4), 3-14. https://doi.org/10/cfs $74 f$

Agnoli, F., Wicherts, J. M., Veldkamp, C. L. S., Albiero, P., \& Cubelli, R. (2017). Questionable research practices among italian research psychologists. PLOS ONE, 12(3), e0172792. https://doi.org/10/f9twdr

Dechartres, A., Ravaud, P., Atal, I., Riveros, C., \& Boutron, I. (2016). Association between trial registration and treatment effect estimates: A meta-epidemiological study. $B M C$ Medicine, 14(1). https://doi.org/10/gf4nmf

Dwan, K., Gamble, C., Williamson, P. R., Kirkham, J. J., \& the Reporting Bias Group. (2013). Systematic Review of the Empirical Evidence of Study Publication Bias and Outcome Reporting Bias-An Updated Review. PLoS ONE, 8(7), e66844. https://doi.org/10/f496f7

Fraser, H., Parker, T., Nakagawa, S., Barnett, A., \& Fidler, F. (2018). Questionable research practices in ecology and evolution. PLOS ONE, 13(7), e0200303. https://doi.org/10/gdtmg2

Goldacre, B., Drysdale, H., Dale, A., Milosevic, I., Slade, E., Hartley, P., Marston, C., Powell-Smith, A., Heneghan, C., \& Mahtani, K. R. (2019). COMPare: A prospective cohort study correcting and monitoring 58 misreported trials in real time. Trials, 20(1), 118. https://doi.org/10/gfvpv9 
Haven, T. L., Errington, T. M., Gleditsch, K. S., van Grootel, L., Jacobs, A. M., Kern, F. G., Piñeiro, R., Rosenblatt, F., \& Mokkink, L. B. (2020). Preregistering Qualitative Research: A Delphi Study. International Journal of Qualitative Methods, 19, 160940692097641. https://doi.org/10.1177/1609406920976417

John, L. K., Loewenstein, G., \& Prelec, D. (2012). Measuring the Prevalence of Questionable Research Practices With Incentives for Truth Telling. Psychological Science, 23(5), 524-532. https://doi.org/10/f33h6z

Kaplan, R. M., \& Irvin, V. L. (2015). Likelihood of Null Effects of Large NHLBI Clinical Trials Has Increased over Time. PLoS ONE, 10(8), e0132382. https://doi.org/10/gc8z6n

Makel, M. C., Hodges, J., Cook, B. G., \& Plucker, J. (2019). Questionable and Open Research Practices in Education Research [Preprint]. EdArXiv. https://doi.org/10.35542/osf.io/f7srb

Nosek, B. A., \& Lakens, D. (2014). Registered Reports. Social Psychology, 45(3), 137-141. https://doi.org/10/gffnpc

Nosek, B. A., Alter, G., Banks, G. C., Borsboom, D., Bowman, S. D., Breckler, S. J., Buck, S., Chambers, C. D., Chin, G., Christensen, G., Contestabile, M., Dafoe, A., Eich, E., Freese, J., Glennerster, R., Goroff, D., Green, D. P., Hesse, B., Humphreys, M., ... Yarkoni, T. (2015). Promoting an open research culture. Science, 348(6242), 1422-1425. https://doi.org/10/gcpzwn

O’Boyle, E. H., Banks, G. C., \& Gonzalez-Mulé, E. (2017). The Chrysalis Effect: How Ugly Initial Results Metamorphosize Into Beautiful Articles. Journal of Management, 43(2), 376-399. https://doi.org/10/gf2k59

Schäfer, T., \& Schwarz, M. A. (2019). The Meaningfulness of Effect Sizes in Psychological Research: Differences Between Sub-Disciplines and the Impact of Potential Biases. Frontiers in Psychology, 10. https://doi.org/10/gfzirr

Silberzahn, R., Uhlmann, E. L., Martin, D. P., Anselmi, P., Aust, F., Awtrey, E., Bahník, Š., Bai, F., Bannard, C., Bonnier, E., Carlsson, R., Cheung, F., Christensen, G., Clay, R., Craig, M. A., Dalla Rosa, A., Dam, L., Evans, M. H., Flores Cervantes, I., ... Nosek, B. A. (2018). Many Analysts, One Data Set: Making Transparent How Variations in Analytic Choices Affect Results. Advances in Methods and Practices in Psychological Science, 1(3), 337-356. https://doi.org/10.1177/2515245917747646 